Tropical Journal of Pharmaceutical Research September 2014; 13 (9): 1463-1470

ISSN: $1596-5996$ (print); 1596-9827 (electronic)

(c) Pharmacotherapy Group, Faculty of Pharmacy, University of Benin, Benin City, 300001 Nigeria.

All rights reserved.

Available online at http://www.tjpr.org

Original Research Article

http://dx.doi.org/10.4314/tjpr.v13i9.12

\title{
Natural Borneol Recycling from Cinnamomum camphor chvar. Borneol Oil Residue by Fractional Distillation and Recrystallization
}

\author{
Xiao Ying Chen ${ }^{1}$, Xiao Ning Zhao ${ }^{2}$, Hui Fang Zeng ${ }^{2}$, Jian Hui Xie ${ }^{1,3}$, Xiao Lu \\ $\mathrm{Chen}^{2}$, Yong Zhuo Liang ${ }^{1}$, Qi Duan $\mathrm{Wu}^{2}$, Zi Ren Su${ }^{1,5}$, Hong Feng Wang ${ }^{4 *}$ and \\ Xiao Ping Lai ${ }^{1,5 *}$ \\ ${ }^{1}$ School of Chinese Materia Medica, Guangzhou University of Chinese Medicine, Guangzhou 510006, ${ }^{2}$ The First Affiliated \\ Hospital of Chinese Medicine, Guangzhou University of Chinese Medicine, Guangzhou 510405, ${ }^{3}$ The Second Affiliated Hospital \\ of Chinese Medicine, Guangzhou University of Chinese Medicine, Guangzhou 510405, ${ }^{4}$ Biotechnology Division, Guangdong \\ Academy of Forestry, Guangzhou, ${ }^{5}$ Dongguan Mathematical Engineering Academy of Chinese Medicine, Guangzhou \\ University of Chinese Medicine, Dongguan, 523808, China
}

*For correspondence: Email: Ixp88@gzucm.edu.cn, wanghf@sinogaf.cn; Tel: +86-20-39358517; Fax: +86-20-39358390

Revised accepted: 7 August 2014

\begin{abstract}
Purpose: To establish an efficient method to recycle natural borneol from Cinnamomum camphor chvar. Borneol oil residue.

Methods: The fractions and raffinate of the oil residue of Cinnamomum camphor chvar. Borneol were obtained by fractional distillation, and analyzed by gas chromatography with flame ionization detector (GC-FID) and gas chromatography- mass spectrometry (GC-MS). Natural borneol was purified by recrystallization and suction filtration. Fractional distillation and recrystallization parameters, including reflux ratio, solvent and solvent amount, were optimized. The physicochemical properties of natural borneol were determined by melting point and optical rotation techniques.

Results: The total yield of natural borneol was $7.6 \%$ under the optimal conditions, viz, reflux ratios of 3:1 and 1:1, with $n$-hexane as recrystallizing solvent. The purity of recrystallized natural borneol was > $99 \%$ at the solvent ratio of 1:1.8 (natural borneol crystal : $n$-hexane). The optical rotation $\left([\alpha]^{24} D=+37^{\circ}\right)$ and melting point $\left(207^{\circ} \mathrm{C}\right)$ of natural borneol were in accord with those of the standard.

Conclusion: These findings indicate that the developed fractional distillation and recrystallization method can be applied to recycle natural borneol from C. camphor chvar. Borneol oil residue.
\end{abstract}

Keywords: Cinnamomum camphora chvar. Borneol, Oil residue, Natural borneol, Fractional distillation, Recrystallization

Tropical Journal of Pharmaceutical Research is indexed by Science Citation Index (SciSearch), Scopus, International Pharmaceutical Abstract, Chemical Abstracts, Embase, Index Copernicus, EBSCO, African Index Medicus, JournalSeek, Journal Citation Reports/Science Edition, Directory of Open Access Journals (DOAJ), African Journal Online, Bioline International, Open-J-Gate and Pharmacy Abstracts

\section{INTRODUCTION}

Cinnamomum camphora chvar. Borneol, a precious and rare medicinal material in Lauraceae family, is discovered in Jiangxi Province and cultivated in many provinces in south China [1]. Due to richest content in natural borneol, the leaves and branches of $C$. camphora chvar. Borneol are industrially applied to extract essential oil for further natural borneol production [2]. Natural borneol (Fig 1) has been widely used in food, medicine, cosmetics, and other industries [3]. To date, extensive efforts have been devoted to the elucidation of chemical components of C. camphora chvar. Borneol essential oil [4] and its oil residue [5,6]. However, 
few of them had ever targeted recycling natural borneol from its oil residue. Steam distillation is widely used in the extraction of essential oil from the leaves and branches of $C$. camphora chvar. Borneol in industry [7]. The essential oil was slowly crystallized. After filtration, the pure natural borneol was separated. However, during this process, a mass of oil residue with natural borneol over $23 \%$ was inevitably generated, which is usually to be discarded or merely used as cheap raw materials and spices $[5,8]$. Hence, oil residue might also be an important source for production of natural borneol. The aim of this study was to investigate the utilization of oil residue for preparation of high-purity natural borneol by virtue of fractional distillation and recrystallization.

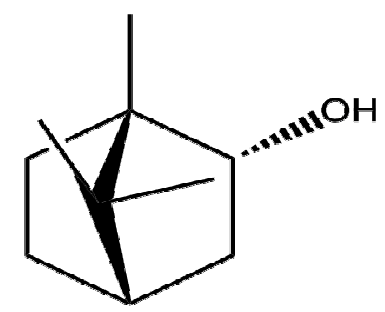

Figure 1: Chemical structure of natural borneol

\section{EXPERIMENTAL}

\section{Materials}

C. camphora chvar. Borneol was collected from the Institute of Forestry Sciences of Ji'an Prefecture of Jiangxi Province (China) in April 2013, and authenticated by Prof. Zi Ren Su at School of Chinese Materia Medica, Guangzhou University of Chinese Medicine, where a voucher specimen (no. 2013122901-09) was deposited in the herbarium. Steam distillation and crystallization were used for the extraction of essential oil from the leaves of $C$. camphora chvar. Borneol and production of natural borneol, respectively. The resulting oil residue during this process was employed in the study. Standard borneol was purchased from Sigma-Aldrich Co, Ltd (Shanghai, China). n-hexane and ethyl acetate were from Guangzhou Chemical Reagents Factory, China.

\section{Apparatus}

The rectification was carried out with a fractionating tower consisting of a temperature control heating jacket, a round-bottom flask (1000 mL), a distillation column $(150 \times 25 \mathrm{~mm}$ inner Diameter) filled with stainless steel Dixon ring packing $(3 \times 3 \mathrm{~mm}$ inner Diameter $)$, an intelligent thermostat, a reflux condenser and receiving flasks. The reflux ratio $(R)$ is the ratio of reflux flow $(\mathrm{L})$ to distillate flow $(\mathrm{D})$, measuring the amounts of volatile oil going up the top of the column and returned back to the column as reflux.

\section{Fractional distillation and recrystallization procedure}

The oil residue $(300 \mathrm{~g})$ was brought to boil in the round-bottom flask of fractionating tower, on condition that the pressure in this system has been kept under normal atmospheric pressure. The oil residue was heated at $170{ }^{\circ} \mathrm{C}$ until refluxing, and then maintained at total reflux for 1 $h$ in order to reach vapor-liquid equilibrium condition. The heater was turned on and the temperature in the fractionating column was monitored. Fractions were collected at various boiling point ranges. Then the reflux ratio was increased to 3 (for $2 \mathrm{~h}$ ) and finally decreased to 1. The experiment should not be stopped until the temperature of the bottom of tower surpassed $210.0{ }^{\circ} \mathrm{C}$. In this experimental protocol, the fractions of oil residue were collected at regular intervals of $20 \mathrm{~min}$, quantified and analyzed by GC-FID and GC-MS respectively. The process flow diagram for fraction distillation and recrystallization of natural borneol from oil residue is shown in Figure 2 . According to respective distilled-off temperature, the distillation process generated three fractions: fraction $A$ (containing natural borneol $\leq 1 \%$ by weight), fraction $\mathrm{B}$ (natural borneol $\leq 10 \%$ by weight) and raffinate (product fraction). Afterwards, the raffinate was stored and crystallized at $4{ }^{\circ} \mathrm{C}$ for $6 \mathrm{~d}$, and then recrystallized. Natural borneol crystals were obtained after suction filtration. The fractional distillation and recrystallization parameters, including reflux ratio, solvent and solvent amount, were optimized.

\section{GC-FID analysis}

Quantitative analysis of the fractions were performed by a flame ionization gas chromatography (FID), using Varian-3900 equipment (Varian Corporation, Palo Alto, CA, USA). The column used was a ZB-WAX, $30 \mathrm{~m} \times$ $0.32 \mathrm{~mm}$ ID $\times 0.25 \mu \mathrm{m}$ film thickness (Phenomenex Corporation, Torrance, CA, USA); nitrogen was the carrier gas at a flow rate of 1 $\mathrm{mL} / \mathrm{min}$; the inlet mode was split 1:20; the injector temperature was $280{ }^{\circ} \mathrm{C}$. The temperature program was as follows: the temperature was initially set at $60^{\circ} \mathrm{C}$, and subsequently increased to $90{ }^{\circ} \mathrm{C}$ at $10^{\circ} \mathrm{C} / \mathrm{min}$, held isothermal for $5 \mathrm{~min}$, then at $3^{\circ} \mathrm{C} / \mathrm{min}$ to 160 ${ }^{\circ} \mathrm{C}$, and then it was raised to $240{ }^{\circ} \mathrm{C}$ at 


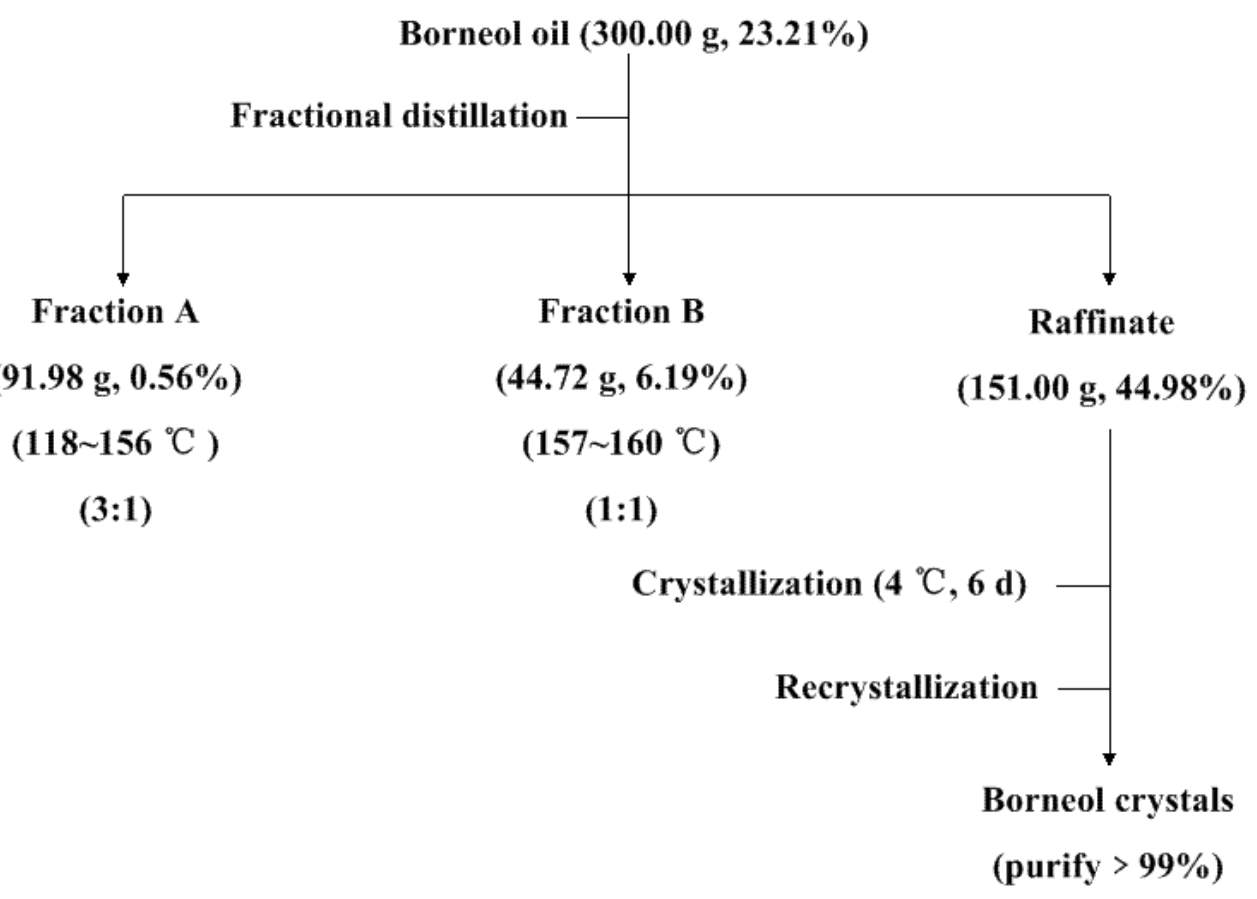

Figure 2: Separation and purification procedure for natural borneol. The yield (weight of oil residue, fraction and crystals, concentration of natural borneol) was shown directly under the fraction, raffinate and crystals. Finally, the crude natural borneol crystals were recrystallized twice to obtain refined natural borneol (purity over $99 \%$ )

$30^{\circ} \mathrm{C} / \mathrm{min}$. Thereafter, this condition was held for $3 \mathrm{~min}$. The injection volume was $1 \mu \mathrm{L}$. Identification of constituents was based on the retention time compared with the standards under identical experimental conditions.

\section{GC-MS analysis}

The GC-MS analysis was performed on an Agilent 6890-5973 model gas chromatographmass spectrometer (Agilent Corporation, Santa Clara, CA, USA). The column was equipped with a HP-5MS (crosslinked $5 \%$ Phenyl Methyl Silox) capillary column $(30 \mathrm{~m} \times 250 \mu \mathrm{m} \times 0.25 \mu \mathrm{m}$ film thickness). The operation parameters were as described above for GC-FID, except for using helium as a carrier gas. The GC-MS analysis was conducted at $70 \mathrm{eV}$ with a mass range of 40 - $400 \mathrm{~m} / \mathrm{z}$ and a scanning velocity of $1494 \mathrm{amu} / \mathrm{s}$. The quadrupole and ion source temperature were 150 and $230{ }^{\circ} \mathrm{C}$, respectively.

\section{Physicochemical properties}

One gram natural borneol crystal was dissolved in $10 \mathrm{~mL}$ dehydrated alcohol to obtain the final concentration of $0.1 \mathrm{~g} / \mathrm{mL}$. The special rotation of the solution was determined with a polarimeter (model 341, Perkin Elmer, USA). Melting point was determined by a melting point apparatus using capillary tubes (model B-545, Büchi, Switzerland).

\section{Statistical analysis}

SPSS Statistics 17.0 was used for statistical analysis. The data were presented as mean \pm standard deviation. The statistical significances within a parameter were evaluated by analysis of variance (ANOVA) followed by least significant difference (LSD) test. Values were considered significantly different at $p<0.05$.

\section{RESULTS}

\section{Separation and purification of natural borneol}

The weight and natural borneol content of all fractions described above are shown in Figure 2. By fractional distillation, oil residue $(300.00 \mathrm{~g}$, $23.21 \%)$ was separated into fraction A $(91.98 \mathrm{~g}$, $0.56 \%)$, fraction B (44.72 $\mathrm{g}, 6.19 \%)$ and raffinate $(151.00 \mathrm{~g}, 44.98 \%)$. Then raffinate was crystallized at $4{ }^{\circ} \mathrm{C}$ to obtain crude natural borneol crystals $(55.00 \mathrm{~g}, 73.6 \%)$. Thereafter high-purity natural borneol $(23.00 \mathrm{~g}, 99 \%)$ was obtained by recrystallization. The total yield of natural borneol by fractional distillation and recrystallization was $7.6 \%$. Natural borneol obtained in this way was white crystalline. Meanwhile, the purified borneol were determined by polarimeter, and the mean optical rotation $[\alpha]^{24}$ D was $+37^{\circ}$ (ethanol, $20^{\circ} \mathrm{C}$ ), which showed that the purified borneol was a dextroisomer. The melting point of natural borneol was $207^{\circ} \mathrm{C}$. 
These parameters were fairly in accord with the quality standard of Chinese Pharmacopoeia [9]. From the foregoing results, fractional distillation coupled with recrystallization might be applied to recycle natural borneol from oil residue.

\section{The chemical composition of all fractions}

To investigate the process of isolation and purification of natural borneol from oil residue, the chemical compositions of the fractions and natural borneol crystals were analyzed by GCMS. The total ion current (TIC) chromatograms of oil residue, fraction ( $A, B$ and raffinate) and natural borneol crystals are shown in Figure 3. Fraction A was rich in a-pinene (Peak 1) which accounted for the percentage of $22.1 \%$. Compared with fraction $A$, the amount of $\alpha$ pinene decreased to $1.60 \%$ in fraction $B$, while the purity of camphor (Peak 2) was raised from $0.50 \%$ to $6.23 \%$, and the content of natural borneol (Peak 3) increased from $0.56 \%$ to 6.19 $\%$. The raffinate (Figure 3D) mainly consisting of natural borneol (44.98\%), bornyl acetate (18.12 $\%)$ and champhor (15.33\%) was subjected to crystallization at $4{ }^{\circ} \mathrm{C}$ for natural borneol. However, the purity of natural borneol obtained after first crystallization was relatively low. Hence further recrystallization was needed. In the present work, the crude sample of borneol was further purified in $\mathrm{n}$-Hexane. As shown in Figure $3 \mathrm{E}$, results indicated that the purity of natural borneol recrystallized twice was higher than 99 $\%$, hence recrystallization was a favorable choice in the preparation of high-purity borneol.

\section{Recovery of natural borneol in oil residue}

Reflux ratio is an important parameter in distillation design and operation. Recovery of natural borneol and separation time were investigated according to different reflux ratios. The results are given in Table 1. As the reflux ratios increased from $1: 1,2: 1$ to $3: 1,1: 1$, the yield and recovery of natural borneol were elevated by $4.5 \%(p<0.05)$ and $10.0 \%(p<$ 0.05 ) respectively. However, with the reflux ratios decreasd from $3: 1,7: 1$ to $3: 1,1: 1$, yield and recovery declined significantly by $3.0 \%(p<$ $0.05)$ and $6.9 \%(p<0.05)$. It can be seen from these results that the maximum yield $(18.3 \pm 1.0$ $\%)$ and recovery $(99.4 \pm 0.5 \%)$ were obtained at the reflux ratios of $3: 1$ and $1: 1$. Total separation time at this reflux ratio was $5.5 \mathrm{~h}$. Considering the effectiveness and power consumption, the reflux ratios of $3: 1$ and 1:1 were chosen.

The yield (\%) of natural borneol in oil residue was the weight of crude crystal to the weight of oil residue. The recovery (\%) was the concentration of natural borneol in raffinate to that in C. camphora chvar. Borneol oil residue. Treatment meant with the same letter was not significantly different from each other at $p>0.05$.

\section{Recrystallization yield and recovery of natural borneol}

The selection of an appropriate solvent is of vital importance in the recrystallization process. In order to investigate which solvent was more suitable, ethyl acetate and n-hexane were chosen. In this experiment, $1 \mathrm{~g}$ crude natural borneol were dissolved in $2 \mathrm{~mL}$ ethyl acetate and $3 \mathrm{~mL}$-hexane to obtain high purity natural borneol by recrystallization, respectively. The results are shown in Table 2. It was suggested that the purity, yield and recovery of natural

Table 1: Effects of different reflux ratios on yield and recovery of natural borneol in oil residue

\begin{tabular}{lccc}
\hline Reflux ratio & Separation time (h) & Yield (\%) & Recovery (\%) \\
\hline $1: 1^{\mathrm{A}}, 2: 1^{\mathrm{B}}$ & 5.0 & $13.8 \pm 0.5^{\mathrm{a}}$ & $89.4 \pm 1.2^{\mathrm{a}}$ \\
$3: 1,7: 1$ & 8.5 & $15.3 \pm 1.1^{\mathrm{D}}$ & $92.5 \pm 1.5^{\mathrm{b}}$ \\
$3: 1,1: 1$ & 5.5 & $18.3 \pm 1.0^{\mathrm{C}}$ & $99.4 \pm 0.5^{\mathrm{C}}$ \\
$5: 1,3: 1$ & 7.0 & $16.9 \pm 0.5^{\mathrm{bc}}$ & $95.7 \pm 1.4^{\mathrm{a}}$ \\
\hline
\end{tabular}

Note: ${ }^{A}$ The reflux ratio as fraction $A$ distilled off; ${ }^{B}$ The reflux ratio as fraction $B$ distilled off

Table 2: Recrystallization of natural borneol in ethyl acetate and $n$-hexane

\begin{tabular}{lcccc}
\hline Solvent & $\begin{array}{c}\text { Material/solvent } \\
\text { ratio }\end{array}$ & Contents (\%) & Yield (\%) & Recovery (\%) \\
\hline Ethyl acetate & $1: 2$ & $83.4 \pm 0.6^{\mathrm{a}}$ & $67.2 \pm 1.8^{\mathrm{a}}$ & $76.1 \pm 1.4^{\mathrm{a}}$ \\
$n$-Hexane & $1: 3$ & $85.9 \pm 1.3^{\mathrm{b}}$ & $76.5 \pm 1.3^{\mathrm{b}}$ & $89.3 \pm 1.2^{\mathrm{b}}$ \\
\hline
\end{tabular}

Note: Material/solvent ratio is the ratio required to dissolve the crude natural borneol 

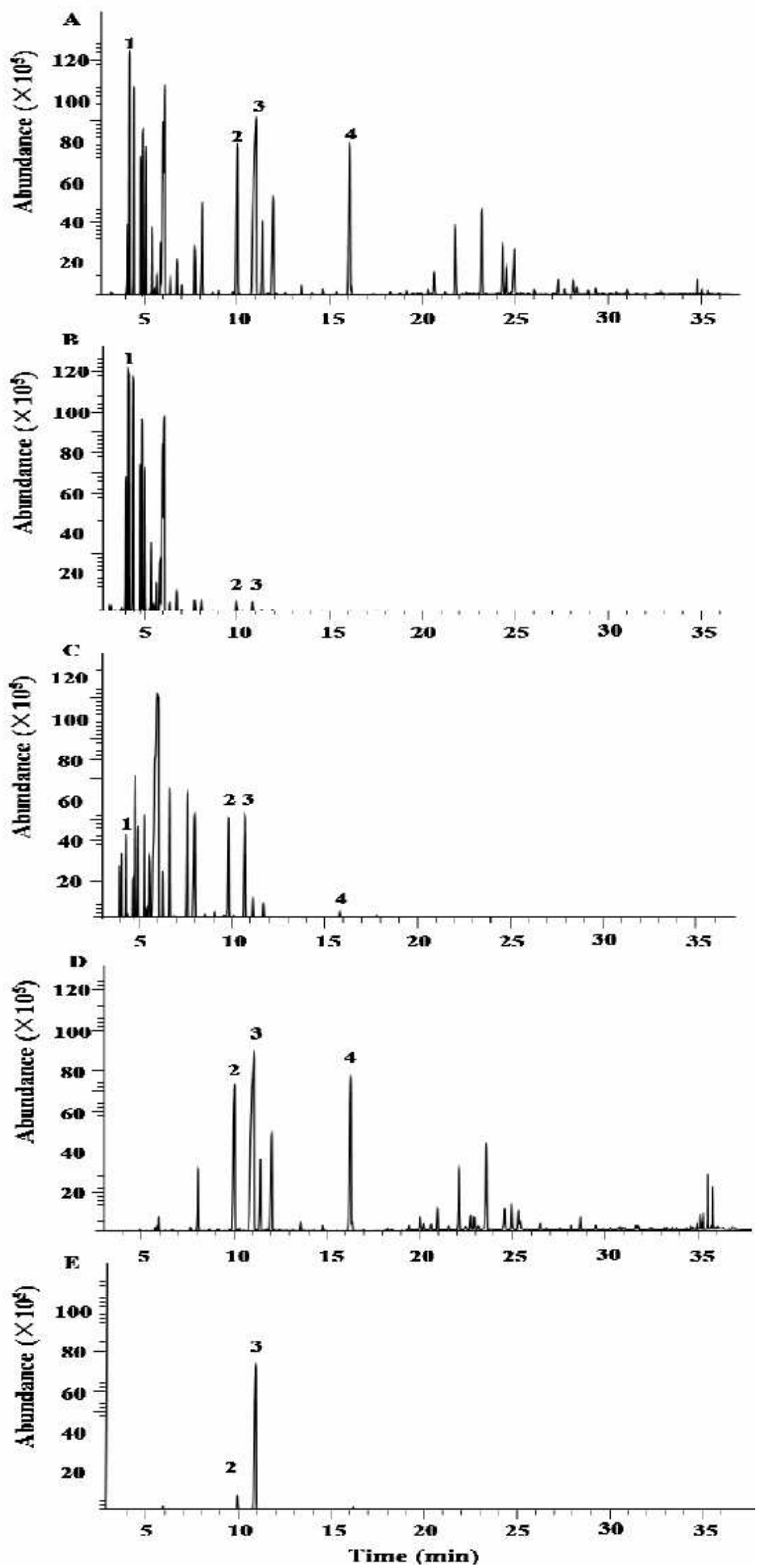

Figure 3: Typical GC-MS chromatograms: $(A)$ oil residue, $(B)$ fraction $A,(C)$ fraction $B$, (D) raffinate; (E) purified natural borneol. Peak identification: (1) $\alpha$-pinene, (2) camphor, (3) natural borneol, (4) bornyl acetate 
borneol crystallized from n-hexane were higher than that from ethyl acetate $(p<0.05)$. Consequently, n-hexane might be superior to ethyl acetate as the recrystallization solvent for natural borneol purification.

The yield (\%) of natural borneol was the weight of refined natural borneol to the crude natural borneol. The recovery (\%) was the concentration of natural borneol in refined crystals to the concentration of crude natural borneol. Treatment meant with the same letter was not significantly different from each other at $p>0.05$.

\section{Effect of solvent on natural borneol crystallizing}

In order to find the favorable n-hexane ratios for natural borneol crystallizing, five different ratios namely $1: 1.2,1: 1.5,1: 1.8,1: 2.0$ and $1: 2.2$ were investigated (Table 3 ). The results showed that the content of natural borneol was elevated and the yield and recovery declined when $n$-hexane ratios rose from $1: 1.2$ to $1: 2.2$. As the $n$-hexane ratio increased from $1: 1.8$ to $1: 2.2$, the yield and recovery decreased obviously $(p<0.05)$. However, the purity of natural borneol was elevated only by $0.5 \%(p>0.05)$. Considering the treatment effect and solvent consumption, nHexane ratio of $1: 1.8$ was chosen as the optimum solvent for recrystallizing natural borneol.

\section{DISCUSSION}

According to the Chinese Pharmacopoeia, borneol is an ingredient of over 60 herbal products [9]. It is widely accepted that synthetic borneol is more toxic than natural borneol. Therefore, a strategy to avoid the toxic effects is to replace synthetic borneol with natural borneol in different borneol products, and the new sources for natural borneol would provide this opportunity [10]. The essential oil obtained by hydrodistillation of young leaves of $C$. camphora chvar. Borneol was richest in natural borneol with content over $66.8 \%$ [11]. The essential oil derived from the leaves was subjected to crystallization. After filtration, the pure natural borneol was separated from oil residue in this process. Reports have revealed that the content of natural borneol in oil residue was still over 23 $\%$ and deserved further exploration $[5,8]$. In this work, fractional distillation combined with recrystallization allowed natural borneol to be obtained with the yield $7.6 \%$ and purity $99 \%$ from oil residue. Moreover, natural borneol product obtained by this method met the quality standard of the Chinese Pharmacopoeia [9].
Therefore, the established regime might serve as an effective alternative for the production of highpurity natural borneol.

Fractional distillation, is a highly efficient and solvent-free separation process which depends on the differences of components' boiling points. As shown in the total ion current (TIC) chromatogram of oil residue (Figure $3 \mathrm{~A}$ ), components of oil residue such as $\alpha$-pinene, limonene and linalool were lower than that of natural borneol. Consequently, these components could be distilled prior to natural borneol, divided into fraction $A$ and $B$, and then the raffinate consisting mainly of natural borneol obtained.

However, as TIC chromatogram displayed, there were some component such as camphor (Peak 2) which had similar molecular weights and boiling points as natural borneol difficult to be separated by fractional distillation. Column chromatography method was previously applied to isolate natural borneol, however, the process consumed large amount of solvents and the yield and recovery were also low [8]. In this work, recrystallization of crude natural borneol crystal was carried out with n-hexane, and natural borneol was achieved with purity over $99 \%$.

Reflux ratio is a crucial parameter in distillation design and operation, which has an important impact on the separation effect and the distillation cost [12]. The purity of target constituent can be improved by increasing the reflux ratio when quantity of column plate is fixed. However, the increasing reflux ratio will push up the energy cost [13]. In this study, the appropriate reflux ratio was investigated. As the reflux ratio increased, more liquid rich in more volatile components was recycled back into the column, and the energy consumption was in positive correlation with the separation time. Results showed that the yield and recovery of natural borneol in raffinate were higher and the separation time was shorter, when fraction $A$ and fraction $B$ were distilled off at the reflux ratios of $3: 1$ and $1: 1$, respectively.

The yield was about $3 \%$ and was higher than that at reflux ratios of $3: 1$ and $7: 1$, indicating that the yield increased as reflux ratio decreased. This might be associated with the dehydrogenation from natural borneol to camphor under long-time high temperature heat. However, precise mechanism deserved further investigation. Natural borneol represented 44.98 $\%$ of the raffinate after reflux. The relatively higher concentration of the target compound in raffinate reduced the difficulty in further process. 
Table 3: First recrystallization of natural borneol in various solvent ratios

\begin{tabular}{llll}
\hline $\boldsymbol{n}$-Hexane ratio & Content (\%) & Yield (\%) & Recovery (\%) \\
\hline $1: 1.2$ & $85.9 \pm 0.8^{\mathrm{a}}$ & $76.5 \pm 0.9^{\mathrm{a}}$ & $89.3 \pm 0.5^{\mathrm{a}}$ \\
$1: 1.5$ & $89.3 \pm 1.0^{\mathrm{b}}$ & $69.1 \pm 1.0^{\mathrm{b}}$ & $83.8 \pm 0.6^{\mathrm{b}}$ \\
$1: 1.8$ & $93.8 \pm 1.2^{\mathrm{c}}$ & $65.6 \pm 0.6^{\mathrm{c}}$ & $83.6 \pm 0.3^{\mathrm{b}}$ \\
$1: 2.0$ & $94.1 \pm 1.6^{\mathrm{c}}$ & $58.1 \pm 0.8^{\mathrm{d}}$ & $74.3 \pm 1.0^{\mathrm{c}}$ \\
$1: 2.2$ & $94.3 \pm 0.6^{\mathrm{c}}$ & $50.0 \pm 1.0^{\mathrm{e}}$ & $64.1 \pm 0.9^{\mathrm{d}}$ \\
\hline
\end{tabular}

Treatments with the same superscript letter were not significantly different from each other at $p>0.05$

Based on the experimental results, the reflux ratios of $3: 1$ and $1: 1$ were chosen in order to obtain the highest yields of natural borneol.

The selection of a suitable solvent is a critical part of the recrystallization procedure [14]. Natural borneol is a small lipophilic compound and freely soluble in ethanol, chloroform and ethyl acetate. Song et al. [6] used gasoline to crystallize natural borneol with the recovery 68 $\%$. He et al [8] applied gasoline and ethyl acetate to purify natural borneol, and the recoveries were $70 \%$ and $57 \%$, respectively. n-Hexane is a major constituent of gasoline, and widely used as cheap and relatively safe solvent in the extraction and refining process. However, there is no report on the purification of natural borneol using $n$ hexane as recrystallization solvent. Therefore, nhexane and ethyl acetate were investigated in this work. The crude crystals were fairly soluble in the boiling $n$-hexane and only sparingly soluble in n-hexane at room temperature. However, impurities were quite soluble in the solvent at room temperature. In this way, when the solvent was cooled and the natural borneol crystallized out, any soluble impurities remained were removed by filtration. Based on the results obtained, n-hexane might be a more appropriate solvent for crystallizating natural borneol from $C$. camphora chvar. Borneol oil residue due to higher purity, yield and recovery.

Solvent amount is another factor affecting recrystallization efficiency [15]. As showed in Table 2, the content of natural borneol increased from $85.9 \pm 0.8 \%$ to $94.3 \pm 0.6 \%$, with respect to the $n$-hexane ratio of 1.2 to 2.2 . When the $n$ hexane ratio was $1: 1.8$, the purity of natural borneol was $93.8 \pm 1.2 \%$, and the yield and recovery were $65.6 \pm 0.6 \%$ and $83.6 \pm 0.3 \%$, respectively. However, as the $n$-hexane ratio increased from 1:1.8 to 1:2.2, the yield and recovery decreased significantly $(p<0.05)$. Hence, the $n$-hexane ratio of $1: 1.8$ was employed.

\section{CONCLUSION}

Fractional distillation followed by recrystallization is an efficient method for recycling natural borneol from Cinnamomum camphor chvar. Borneol oil residue. The proposed method is economical and practical, in terms of resource optimization, with relatively higher purity, yield and recovery. Hence, it is potentially an effective option for the production of high-purity natural borneol and should also suitable for industrial scale-up.

\section{ACKNOWLEDGEMENT}

This work was supported by grants from Guangdong Provincial Natural Science Foundation Team (no. S2012030006598), Science and Technology Major Project of Guangdong Province (no. 2012A080202002), National Science and Technology Support Program (no. 2012BAI29B00), Combined Program of Ministry of Education of Guangdong Province (no. 2012B090600007), Hong Kong, Macao and Taiwan Special Science and Technology Cooperation Program (no. 2014DFH30010). Xiao Ying Chen, Xiao Ning Zhao and Hui Fang Zeng equally contributed to this work.

\section{REFERENCES}

1. Chen HM, Sun LF. The Exploitation and Utilization Prospects of Borneol Cinnamomum camphora Resources in Ji'an, Jiangxi Province. Sci Silva Sin 2006; (3): 94-98.

2. Wu ML, Chen XL, Liao ZX, Peng ZL, Zhou RW, Gan $Q$. Research utilization and prospect of borneol Camphor. Jiangxi Forest Sci and Technol 2011; (2): 30-36.

3. Zhao JY, Lu Y, Du SY, Song X, Bai J, Wang $Y$, Comparative pharmacokinetic studies of borneol in mouse plasma and brain by different administrations. $J$ Zhejiang Univ Sci b 2012; 13(12): 990-996.

4. Chen XL, Zeng HG, Xie ZP, Liu Z, Guo YL, Liao ZX, OilYield and Chemical Constituents of Essential Oils Steam-distilled from Leaves of Cinnamomum 
camphora chvar.Borneol at Different Time Section Jiangxi Forest Sci and Technol 2011; (3): 1-2.

5. Zhang $T$, Jiang $X M, X u H N$, Chemical Components Identification of Waste Essential Oil during Borneol Refining by GC-MS. Food Res Dev 2012; (11): 145147.

6. Song YF, Luo JL, analysis of residue oil constituents from borneol distillation process. Chem Ind forest Prod 2001; (3): 39-41.

7. Chen $L, S u$ J, Li L, Li B, Li W, A new source of natural $D$ borneol and its characteristic. J Med Plants Res 2011; (5): 3440-3447.

8. He HC, Chen QW, Deng LY, Optimization of the purification process of natural borneol. Hunan Forest Sci \& Technol 2013; (3): 14-17.

9. The State Pharmacopoeia Commission of the P. R. China, Pharmacopoeia of the People's Republic of China (Vol.1), Beijing: 2010, Chemical Industry Press; pp 42, 373.

10. Jiang XF, Zou JL, Mei YY, Law FC, Qiao YJ, Yao MC, Preliminary study: biotransformation of borneol to camphor in mice, rats, and rabbits, World Sci \& Technol 2008; 10(3): 27-36.
11. Shi SJ, Wu QD, Su JY, Li CW, Zhao XN, Xie JX, Cui SH, Su ZR, Zeng HF, Composition analysis of volatile oils from flowers, leaves and branches of Cinnamomum camphora chvar. Borneol in china. J Essent Oil Res 2013; 25(5): 395-401.

12. Tavan $Y$, Hosseini SH, Design and simulation of a reactive distillation process to produce high-purity ethyl acetate. J Taiwan Inst Chen E 2013 44(4): 577585.

13. Lim KT, Matsumoto $H$, Yamaki T, Matsuda K, A Framework for Application of Genetic Algorithm to Model-Based Design of Reactive Distillation Process. J Chem Eng Jpn 2014; 47: 187-194.

14. Liu G, Wang $H$, Jiang $Y$, Recrystallization and Micronization of Camptothecin by the Supercritical Antisolvent Process: Influence of Solvents. Ind \& Eng Chem Res 2013; (52): 15049-15056.

15. Ye $C$, Zheng $H$, Wu T, Fan M, Feng J, Li W, Optimization of solvent crystallization process in obtaining high purity anthracene and carbazole from crude anthracene. Aiche J 2014; 60(1): 275-281. 